\title{
Hydrodynamic equations of anisotropic, polarized and inhomogeneous superfluid vortex tangles
}

\author{
D. Jou ${ }^{1}$, M.S. Mongiovì ${ }^{2}$ and M. Sciacca ${ }^{2}$ \\ ${ }^{1}$ Departament de Física, Universitat Autònoma de Barcelona, 08193 Bellaterra, Catalonia, Spain \\ 2 Dipartimento di Metodi e Modelli Matematici Università di Palermo, Palermo, Italy
}

Key words: Superfluid Turbulence, Liquid Helium II, Hydrodynamic equations

PACS number(s): 67.25.dk, 47.37.+q, 67.25.dm

\begin{abstract}
We include the effects of anisotropy and polarization in the hydrodynamics of inhomogeneous vortex tangles, thus generalizing the well known Hall-Vinen-Bekarevich-Khalatnikov equations, which do not take them in consideration. These effects contribute to the mutual friction force $\mathbf{F}_{n s}$ between normal and superfluid components and to the vortex tension force $\rho_{s} \mathbf{T}$. These equations are complemented by an evolution equation for the vortex line density $L$, which takes into account these contributions. These equations are expected to be more suitable than the usual ones for rotating counterflows, or turbulence behind a cylinder, or turbulence produced by a grid of parallel thin cylinders towed across a superfluid, because in these situations polarization is expected to play a relevant role.
\end{abstract}

\section{Introduction}

The possibility to use turbulent superfluids to explore with relative facility turbulent flows with high Reynolds numbers is an increasingly exciting perspective. The main attractive issue of superfluid turbulence are quantized vortices, which are filamentous vortices, whose core dimension is of the order of the atomic diameter of the helium atom, of the order of $1 \AA$, due to the rotational of superfluid component whose circulation is quantized. From a microscopic point of view, they are described by specifying the whole detailed curve of each vortex line, but from a macroscopic perspective this detailed description is lost and it is usually reduced to simply a scalar quantity $L$, the vortex line density, i.e., the total length of vortex lines per unit volume.

Quantized vortices in superfluids have been mainly studied in two typical situations: rotating superfluids and counterflow experiments, the latter meaning the presence of a heat flux, but with zero barycentric motion. In these situations the vortices are modeled, respectively, as an array of parallel rectilinear vortices or as an almost isotropic tangle. In both cases, the mutual force between the normal component and the superfluid due to the presence of vortex lines is well known, and the so-called vortex line tension, due to the curvature of vortex lines, is zero [1]- 4].

\footnotetext{
${ }^{0}$ E-mail addresses: david.jou@uab.es (D. Jou), mongiovi@unipa.it (M. S. Mongiovì), msciacca@unipa.it (M. Sciacca)
} 
However, in other situations, as rotating counterflow or non stationary Couette and Poiseuille flows, or behind a grid towed across a quiescent superfluid, one expects a partially polarized vortex tangle, as a compromise between the orienting effect of a rotation or of a macroscopic velocity gradient, and the randomizing effect of the relative velocity of normal and superfluid components. In these cases, the mutual friction force $\mathbf{F}_{n s}$ between these components, as well as the nonvanishing tension of the vortex lines $\rho_{s} \mathbf{T}$, which are fundamental ingredients of the hydrodynamics of turbulent superfluids in the well-known Hall-Vinen-Bekarevich-Khalatnikov (HVBK) model [5, 6], are not sufficiently known. Thus, the exploration of $\mathbf{F}_{n s}$ and $\mathbf{T}$ for partially polarized tangles with nonvanishing average curvature of vortex lines is an open topic.

The quantitative values of the corrections are expected to be relevant in some steady states (as rotating counterflows, or turbulence behind a cylinder, or turbulence produced by a grid of parallel thin cylinders towed across a superfluid) where a sufficiently high polarization may induce macroscopic eddies [7], and in many of the proposed experiments which involve nonstationary flows, for their own sake or in view to the application of classical measurement techniques to superfluids, as for instance thin oscillating wires or small-particle velocimetry. Thus a basic understanding of these effects is essential to obtain the form of $\mathbf{F}_{n s}$ and $\mathbf{T}$. This is the aim of this paper.

The structure of the paper is the following one: in Section 2 an introduction to the usual HVBK model is made, with special emphasis on the expression of the mutual friction force $\mathbf{F}_{n s}$ and the tension $\mathbf{T}$ in the cases of rotating helium and counterflow superfluid turbulence; in Section 3 a microscopic expression for the friction force and vortex tension is given, checking their limit of validity in HVBK model; in Section 4 a more general expression of the HVBK model is given through more exhaustive expressions for the mutual friction force $\mathbf{F}_{n s}$ and tension $\mathbf{T}$. The model is completed by a generalization of the Vinen's equation, as an evolution equation for the vortex line density $L$. At last, in Section 5 an application of the model to the interesting case of rotating counterflow turbulence is made, which takes advance of the recent study on the anisotropy of the vortex tangle.

\section{HVBK model. Evolution equations for the normal and the superfluid components}

It is known that if a superfluid $\left({ }^{4} \mathrm{He}\right.$ and ${ }^{3} \mathrm{He}$ liquid helium, Bose-Einstein condensates, neutron stars, ...) rotates at a constant angular velocity $\Omega$, exceeding a critical value $\Omega_{c}$, an ordered array of quantized vortex lines of equal circulation $\kappa$ parallel to the rotation axis is created [1][4]. The quantum of vorticity is $\kappa=h / m_{4}$ in superfluid ${ }^{4} \mathrm{He}$, with $h$ the Planck constant and $m_{4}$ the mass of ${ }^{4} \mathrm{He}$ atom; in ${ }^{3} \mathrm{He}$ and neutron stars, which are formed by fermionic particles, the superfluidity is due to the formation of Cooper pairs, and therefore it is $\kappa=h / 2 m$, where $m$ is the mass of an ${ }^{3} \mathrm{He}$ atom or of a neutron, respectively.

In most literature, the motion of a superfluid is modeled using Landau's two-fluid model, which regards the fluid to be made of two completely mixed components: the normal fluid and the superfluid, with densities $\rho_{n}$ and $\rho_{s}$ respectively, and velocities $\mathbf{v}_{n}$ and $\mathbf{v}_{s}$ respectively, with total mass density $\rho$ and barycentric velocity $\mathbf{v}$ defined by $\rho=\rho_{s}+\rho_{n}$ and $\rho \mathbf{v}=\rho_{s} \mathbf{v}_{s}+\rho_{n} \mathbf{v}_{n}$. The first component is related to thermally excited states (phonons and rotons) that form a classical Navier-Stokes viscous fluid. The second component is related to the quantum coherent ground state and it is an ideal fluid, which does not experience dissipation neither 
carries entropy.

If the superfluid is put in rotation with angular velocity $\Omega$ higher than $\Omega_{c}$, the ordered array of parallel quantized vortex lines is described by introducing the line density $L$, defined as the average vortex line length per unit volume, equivalent to the areal density of vortex lines, which is proportional to the angular velocity [1]-[4], namely $L=2 \Omega / \kappa$.

It is well known too that a disordered tangle of quantized vortex lines is created in the so-called counterflow superfluid turbulence [1]-4], characterized by no matter flow but only heat transport, exceeding a critical heat flux $q_{c}$. When the turbulence is fully developed, the line density $L$ is proportional to the square of the averaged counterflow velocity vector

$$
\mathbf{V}_{n s}=\left[\mathbf{v}_{n s}\right]_{a v}=\frac{1}{\Lambda} \int \mathbf{v}_{n s} d \Lambda,
$$

related to heat flux $\left(\mathbf{v}_{n s}=\mathbf{v}_{n}-\mathbf{v}_{s}\right.$ being the microscopic counterflow velocity) [1]-[4], $L \simeq$ $\gamma_{H}^{2} \mathbf{V}_{n s}^{2} / \kappa^{2}$, the dimensionless coefficient $\gamma_{H}$ being dependent on the temperature. In (2.1) and in the following, capital letters denote local macroscopic velocities averaged over a small mesoscopic volume $\Lambda$, threaded by a high density of vortex lines.

In this paper we will use two different averages which, for the sake of clarity, we explicit here. The first one is that used in (2.1) where the integration is made over a small volume $\Lambda$, whereas the other one will be made on all vortices contained in the same volume $\Lambda$, that is

$$
<->=\frac{1}{L \Lambda} \int-d \xi
$$

where $L$ is the vortex line density in the volume $\Lambda$ and $\xi$ is the parameter indicating the arc-length along the vortex line, and the integral is made on all vortices contained in $\Lambda$. The two averages are linked by the relation $[-]_{a v}=L<->$.

Due to the smallness of the quantum of circulation, even a relatively weak rotation or a small counterflow velocity produces a large density of vortex filaments. It is therefore possible to develop a set of macroscopic hydrodynamic equations which average over the presence of many individual vortex lines and incorporate the macroscopic effects of the vortices in the evolution equations for superfluid and normal fluid velocities.

As an illustration of the order of magnitude of the values of $L$ in current situations, let us mention that in counterflow in a cylindrical channel the line length density $L$ satisfies the relation $L^{1 / 2}=\gamma V_{n s}-\frac{b}{d}$, where $d$ is the diameter of the channel (see for example [8] and [9]); coefficients $\gamma$ and $b$ assume different values in different turbulent regimes. In particular in [9] it has been shown that, for values of the dimensionless quantity $x=\frac{V_{n s} d}{\kappa}$ greater than 219 at $T=1.5 \mathrm{~K}$ and greater than 186 at $T=1.7 \mathrm{~K}$, the system undergoes a transition from the turbulent TI state, with a low density of vortex lines, to the fully developed turbulent TII state, with a high density of vortex lines. The experimental results shows that, at the transition TI-TII in a volume of $1 \mathrm{~cm}^{3}$ there is a vortex tangle of about total length $\mathcal{L}=400$ $\mathrm{cm}$.

A set of hydrodynamical equations frequently used is the Hall-Vinen-Bekarevich-Khalatnikov (HVBK) model [1, 5, 6], [10]-[14]. These equations, which were derived by a number of researchers over the years, are those of the two-fluid model, modified to incorporate the presence of vortices. Here, for sake of simplicity, we consider the incompressible HVBK equations, which in an inertial frame are written [1, 14]: $\nabla \cdot \mathbf{V}_{n}=0, \nabla \cdot \mathbf{V}_{s}=0$, and

$$
\rho_{n} \frac{\partial \mathbf{V}_{n}}{\partial t}+\rho_{n}\left(\mathbf{V}_{n} \cdot \nabla\right) \mathbf{V}_{n}=-\frac{\rho_{n}}{\rho} \nabla p_{n}-\rho_{s} S \nabla T+\mathbf{F}_{n s}+\eta \nabla^{2} \mathbf{V}_{n}
$$




$$
\rho_{s} \frac{\partial \mathbf{V}_{s}}{\partial t}+\rho_{s}\left(\mathbf{V}_{s} \cdot \nabla\right) \mathbf{V}_{s}=-\frac{\rho_{s}}{\rho} \nabla p_{s}+\rho_{s} S \nabla T-\mathbf{F}_{n s}+\rho_{s} \mathbf{T}
$$

In these equations $p_{n}$ and $p_{s}$ are the effective pressures acting on the normal and the superfluid component, respectively, defined as $\nabla p_{n}=\nabla p+\left(\rho_{s} / 2\right) \nabla V_{n s}^{2}$ and $\nabla p_{s}=\nabla p-\left(\rho_{n} / 2\right) \nabla V_{n s}^{2}, p$ the total pressure, $S$ the entropy, $T$ the absolute temperature, and $\eta$ the dynamic viscosity of the normal component. The effects of the vortices are described by $\mathbf{F}_{n s}$, the friction force exerted by the superfluid component on the normal component - which bears an opposite sign in the evolution equation (2.4) - and $\rho_{s} \mathbf{T}$, the vortex tension force, related to the average curvature of the vortices, and which is a restoring force arising in curved vortex lines, as a consequence of the fact that the streamlines on the side towards the centre of curvature are closer together than in the external side, which makes a difference of pressure between both sides, producing and inward-directed force normal to the filament. Equations (2.3)-(2.4) can be applied when the line length $L$ per unit volume is high, so that the fluctuations become statistically irrelevant.

The expression of the mutual friction force $\mathbf{F}_{n s}$ in the HVBK equations is [14]

$$
\mathbf{F}_{n s}^{(H V B K)}=\rho_{s} \alpha \hat{\omega} \times\left[\vec{\omega} \times\left(\mathbf{V}_{n s}-\tilde{\beta} \nabla \times \hat{\omega}\right)\right]+\rho_{s} \alpha^{\prime} \vec{\omega} \times\left(\mathbf{V}_{n s}-\tilde{\beta} \nabla \times \hat{\omega}\right),
$$

where $\vec{\omega}=\nabla \times \mathbf{V}_{s}$ is the local averaged superfluid vorticity, $\hat{\omega}=\vec{\omega} /|\omega|$ the unit vector along $\vec{\omega}$, and $\tilde{\beta}$ the vortex tension parameter, defined as [1]

$$
\tilde{\beta}=\frac{\epsilon_{V}}{\kappa \rho_{s}}=\frac{\kappa}{4 \pi} \ln \left(\frac{c}{a_{0} L^{1 / 2}}\right),
$$

with $c$ a constant of the order of unity, $a_{0}$ the radius of the vortex core, of about $1 \AA$, and $\epsilon_{V}$ the energy per unit length of vortex line. Coefficients $\alpha$ and $\alpha^{\prime}$ depend on temperature and describe the interaction between the normal fluid and the vortices. They are linked to the well-known Hall-Vinen coefficients $B$ and $B^{\prime}$ by the relations $\alpha=B\left(\rho_{n} / 2 \rho\right), \alpha^{\prime}=B^{\prime}\left(\rho_{n} / 2 \rho\right)$ [1]. Note that this friction force is mediated through the presence of the vortex lines, as $\vec{\omega}$ is related to them.

In (2.4) $\rho_{s} \mathbf{T}$ is the vortex tension force whose microscopic meaning will be discussed in Section 3. In the HVBK equations $\mathbf{T}$ is substituted with

$$
\mathbf{T}^{(H V B K)}=(\tilde{\beta} \nabla \times \hat{\omega}) \times \vec{\omega}=\tilde{\beta}(\vec{\omega} \cdot \nabla) \hat{\omega} .
$$

From this expression it follows that in this approximation $\mathbf{T}$ vanishes when the vorticity is homogeneous $(\nabla \vec{\omega}=0)$, or when the vortex lines are parallel to each other, in which case $\nabla \times \hat{\omega}=0$.

In the regular vortex array produced by the pure rotation, the vorticity $\vec{\omega}$ equals $2 \boldsymbol{\Omega}$ everywhere, $\boldsymbol{\Omega}$ being the angular velocity, and the mutual friction force $\mathbf{F}_{n s}(2.5)$ assumes the Hall-Vinen expression

$$
\mathbf{F}_{n s}=2 \rho_{s} \alpha \hat{\boldsymbol{\Omega}} \times\left[\boldsymbol{\Omega} \times\left(\mathbf{V}_{n}-\mathbf{V}_{s}\right)\right]+2 \rho_{s} \alpha^{\prime} \boldsymbol{\Omega} \times\left(\mathbf{V}_{n}-\mathbf{V}_{s}\right),
$$

because $\nabla \times \vec{\omega}=0$, and $\vec{\omega}=2 \boldsymbol{\Omega}$. As seen in (2.7), the vortex tension $\mathbf{T}$ vanishes, because the vortices are straight lines.

In counterflow superfluid turbulence the vortex tangle is supposed isotropic and the mutual friction force (2.5) is expressed by [1]-[4]

$$
\mathbf{F}_{n s}=-\frac{2}{3} \rho_{s} \kappa \alpha L \mathbf{V}_{n s}
$$


The assumed isotropy of the tangle implies that the vortex tension $\mathbf{T}$ vanishes because the microscopic vorticity would be isotropically distributed, in such a way that the average $\vec{\omega}$ is null everywhere. However, the hypothesis of complete isotropy of the tangle is not confirmed by the simulation of the dynamics of the vortex tangle made by Schwarz [15]-17] and in the experiments [18]. Indeed the particular direction of the counterflow breaks the rotational symmetry in the dynamics. In Section 5 , we will consider in detail this case.

The HVBK equations (2.3)-(2.4) have been used in Refs. [10]-12, 14] to study different experimental situations in liquid He II, as Taylor-Couette flows, end effects in rotating helium and plane Poiseuille flow. Recently, these equations have also been used in Refs [19, 20] to study the jump in the rotational speed observed in neutron stars, whose interior is a neutron superfluid at a huge density. In some cases these equations, with the expressions of $\mathbf{F}_{n s}$ and $\mathbf{T}$, were able to describe, in reasonable qualitative agreement with experiments, the instability of the laminar regime. But, owing to the limitations imposed by the choice (2.5) and (2.7) for $\mathbf{F}_{n s}$ and $\mathbf{T}$, the applications to other situations is not possible or does not produce accord with experiments.

One of the aims of the present paper is the generalization of the expressions (2.5) and (2.7) of $\mathbf{F}_{n s}$ and $\mathbf{T}$, to allow the application of the HVBK equations to other interesting phenomena. First, we will analyze the limit of validity of expressions (2.5) and (2.7) for $\mathbf{F}_{n s}$ and $\mathbf{T}$ in the HVBK model, which, as we will show in the following Section, are restricted to regions with a high density of vortex lines, all pointing in the same direction, and with the same curvature vector. Thus we will pay special attention to the fluctuations in the direction of the vortex lines and to the local anisotropy and polarization of the tangle as well as on its inhomogeneity, and their consequences on $\mathbf{F}_{n s}$ and $\mathbf{T}$.

Another topic addressed in the work is to recognize the necessity to add to equations (2.3) and (2.4) an additional equation for vortex line density $L$. In Subsections 4.2 and 4.3 an evolution equation for $L$ is written, which generalizes Vinen equation to anisotropic, polarized, inhomogeneous situations.

\section{Expressions for $\mathbf{F}_{n s}$ and $\mathbf{T}$}

The HVBK equations (2.3)-(2.4), with $\mathbf{F}_{n s}$ and $\mathbf{T}$ expressed by (2.5) and (2.7), have been used in several problems in superfluid hydrodynamics, for instance, to study the Taylor-Couette flow in helium II [10]-12, 14] and, recently, the jump in the rotational speed observed in neutron stars [19, 20]. The numerical simulations obtained with these equations show the formation of macroscopic vortices, but they do not include the microscopic chaotic dynamics expressed, for instance, by the fluctuations of the tangents to the vortex lines.

Our aim here is to incorporate inhomogeneities, anisotropy, polarization, and tension of the tangle of vortex lines in an extension of the HVBK hydrodynamic equations. To this purpose, we make a critical analysis of these equations, and we perform a suitable modification of them. Furthermore, we include an additional equation for the evolution of $L$, besides the equations (2.3) and (2.4), because $L$ is related to forces and tensions.

\subsection{Microscopic expressions for friction force and vortex tension}

To derive the expressions (2.5) and (2.7) of $\mathbf{F}_{n s}$ and of $\mathbf{T}$, used in the HVBK equations, and to determine their limit of validity, we consider the microscopic description of a vortex tangle in 
the vortex filament model by Schwarz [15]-17]. In this model, a quantized vortex filament is assumed as a classical vortex line in the superfluid with a hollow core and quantized circulation $\kappa$. The vortex line is described by a vectorial function $\mathbf{s}(\xi, t), \xi$ being the arc-length, $\mathbf{s}^{\prime}=\partial \mathbf{s} / \partial \xi$ the unit vector tangent along the vortex line, and $\mathbf{s}^{\prime \prime}=\partial^{2} \mathbf{s} / \partial \xi^{2}$ the curvature vector. The normal component reacts to a moving vortex by producing the "microscopic" mutual friction force $-\mathbf{f}_{M F}$. The microscopic form of this force is directly related to roton scattering from an element of line. The drag force on unit length of line due to scattering of excitations is [1]

$$
\mathbf{f}_{D}=-D \mathbf{s}^{\prime} \times\left[\mathbf{s}^{\prime} \times\left(\mathbf{v}_{R}-\mathbf{v}_{L}\right)\right]+D_{t} \mathbf{s}^{\prime} \times\left(\mathbf{v}_{R}-\mathbf{v}_{L}\right)
$$

where $\mathbf{v}_{R}$ is the roton drift velocity near the core and where the microscopic parameters $D$ and $D_{t}$ are related to the kinetic scattering coefficients $\sigma_{\|}$and $\sigma_{\perp}$ by [1]

$$
D=\rho_{n} v_{G} \sigma_{\|} \quad D_{t}=D^{\prime}-\rho_{n} \kappa=\rho_{n} v_{G} \sigma_{\perp}-\rho_{n} \kappa
$$

where $v_{G}$ is the thermal average group velocity of rotons. The last term in equation (3.2) is due to the presence of the Iordanskii force [21].

Inserting in (3.1) the expression of the vortex line element velocity

$$
\mathbf{v}_{L}=\frac{d \mathbf{s}}{d t}=\mathbf{v}_{s l}+\alpha \mathbf{s}^{\prime} \times\left(\mathbf{v}_{n}-\mathbf{v}_{s l}\right)-\alpha^{\prime} \mathbf{s}^{\prime} \times\left[\mathbf{s}^{\prime} \times\left(\mathbf{v}_{n}-\mathbf{v}_{s l}\right)\right]
$$

where $\mathbf{v}_{s l}=\mathbf{v}_{s}+\mathbf{v}_{\mathbf{i}}$ is the "local superfluid velocity", sum of the superfluid velocity at large distance from any vortex line and of the self-induced velocity — described by the tangent unit vector $\mathbf{s}^{\prime}$ and by the curvature $\mathbf{s}^{\prime \prime}$ - the drag force can be equally described in terms of $\left(\mathbf{v}_{n}-\mathbf{v}_{s l}\right)$ as follows [1]

$$
\mathbf{f}_{M F}=\alpha \rho_{s} \kappa \mathbf{s}^{\prime} \times\left[\mathbf{s}^{\prime} \times\left(\mathbf{v}_{n}-\mathbf{v}_{s l}\right)\right]+\alpha^{\prime} \rho_{s} \kappa \mathbf{s}^{\prime} \times\left(\mathbf{v}_{n}-\mathbf{v}_{s l}\right) .
$$

Coefficients $\alpha$ and $\alpha^{\prime}$ are dimensionless quantities whose link to the kinetic scattering coefficients $D$ and $D^{\prime}$ can be found in Ref. [1].

In the "local induction approximation", that is ignoring contribution on the self-induced velocity coming from the nonlocal portion of the vortex, the self-induced velocity $\mathbf{v}_{\mathbf{i}}$ is approximated by [1]- -4$]$

$$
\mathbf{v}_{\mathbf{i}} \simeq \mathbf{v}_{\mathbf{i}}^{(l o c)}=\tilde{\beta}\left[\mathbf{s}^{\prime} \times \mathbf{s}^{\prime \prime}\right] .
$$

The intensity of $\mathbf{v}_{\mathbf{i}}$ is $\left|\mathbf{v}_{\mathbf{i}}\right| \simeq \tilde{\beta} / R$, with $R$ the curvature radius of the vortex lines. The selfinduced velocity is zero if the vortices are straight lines. Observing that $\mathbf{v}_{n}-\mathbf{v}_{s l}=\mathbf{v}_{n s}-\mathbf{v}_{\mathbf{i}}$, being $\mathbf{v}_{n s}$ the microscopic counterflow velocity, the mutual friction force per unit length assumes the expression

$$
\mathbf{f}_{M F}=\alpha \rho_{s} \kappa \mathbf{s}^{\prime} \times\left[\mathbf{s}^{\prime} \times\left(\mathbf{v}_{n s}-\mathbf{v}_{\mathbf{i}}\right)\right]+\alpha^{\prime} \rho_{s} \kappa \mathbf{s}^{\prime} \times\left(\mathbf{v}_{n s}-\mathbf{v}_{\mathbf{i}}\right) .
$$

In the evolution equations for the velocities of normal and superfluid components, the "macroscopic" mutual friction force $\mathbf{F}_{n s}$ per unit volume which superfluid and normal components mutually exert, is obtained by averaging (3.6) over a small volume $\Lambda$; one has

$$
<\mathbf{f}_{M F}>=\frac{\int \mathbf{f}_{M F} d \xi}{\int d \xi}=\frac{1}{\Lambda L} \int \mathbf{f}_{M F} d \xi
$$

where the integral is made over all the vortices contained in the volume $\Lambda$; to obtain $\mathbf{F}_{n s}$, we must multiply the average (3.7), which denotes the average mutual friction force per unit length, by $L$, which denotes the length of vortex lines per unit of volume. One obtains

$$
\mathbf{F}_{n s}=\left[\mathbf{f}_{n s}\right]_{a v}=L<\mathbf{f}_{M F}>=\alpha \rho_{s} \kappa L<\mathbf{s}^{\prime} \times\left[\mathbf{s}^{\prime} \times\left(\mathbf{v}_{n s}-\mathbf{v}_{\mathbf{i}}\right)\right]>+\alpha^{\prime} \rho_{s} \kappa L<\mathbf{s}^{\prime} \times\left(\mathbf{v}_{n s}-\mathbf{v}_{\mathbf{i}}\right)>.
$$


The vortex tension force $\mathbf{T}$ arises from the microscopic form of the evolution equation of $\mathbf{v}_{s}$. This equation is a consequence of the phase-slip of the superfluid wave function $\varphi$ and, neglecting the mutual friction force, it is written [1], 22]

$$
\frac{\partial \mathbf{v}_{s}}{\partial t}=\mathbf{v}_{L} \times \vec{\omega}_{m i c r}+\nabla \mu
$$

Here $\mathbf{v}_{L}$ is the velocity of the vortex line and the term $\nabla \mu$ describes the effects of pressure and temperature gradients, $\nabla \mu=-\nabla p_{s} / \rho+S \nabla T ; \mathbf{v}_{s}$ denotes the microscopic velocity of the superfluid around the vortex and $\vec{\omega}_{m i c r}=\oint \mathbf{v}_{s} \cdot d \mathbf{x}$ the vorticity of the single vortex line.

In equation (3.9), $\vec{\omega}_{\text {micr }}$ is given and it determines the evolution of $\mathbf{v}_{s}$; when making the average over a volume, $\vec{\omega}_{\text {micr }}$ will become a vector field corresponding to the averages on vortex lines and the corresponding equation will describe the evolution of the averaged superfluid velocity.

Note that $\vec{\omega}_{\text {micr }}$ is a vector tangent to the vortex line which describes the quantized vorticity around a vortex line. On the vortex line itself $\mathbf{v}_{s}$ would be singular, but the rotational is not singular, but is given by the vorticity quantum $\kappa$.

Note that $\mathbf{v}_{L}=\mathbf{v}_{s l}=\mathbf{v}_{s}+\mathbf{v}_{\mathbf{i}}$ [1], with $\mathbf{v}_{\mathbf{i}}$ the "self induced-velocity". When the average on the mesoscopic volume $\Lambda$ is taken one has

$$
\frac{\partial \mathbf{V}_{s}}{\partial t}=\left[\mathbf{v}_{s} \times \vec{\omega}_{m i c r}\right]_{a v}+\left[\mathbf{v}_{\mathbf{i}} \times \vec{\omega}_{m i c r}\right]_{a v}-\frac{\nabla p_{s}}{\rho}+S \nabla T
$$

where $\nabla \mu=-\nabla p_{s} / \rho+S \nabla T$.

In the HVBK equation, one make the approximation $\left[\mathbf{v}_{s} \times \vec{\omega}_{\text {micr }}\right]_{a v}=\mathbf{V}_{s} \times\left(\nabla \times \mathbf{V}_{s}\right)=$ $-\left(\mathbf{V}_{s} \cdot \nabla\right) \mathbf{V}_{s}+(1 / 2) \nabla \mathbf{V}_{s}^{2}$, obtaining

$$
\rho_{s} \frac{\partial \mathbf{V}_{s}}{\partial t}+\rho_{s}\left(\mathbf{V}_{s} \cdot \nabla\right) \mathbf{V}_{s}=\frac{1}{2} \nabla \mathbf{V}_{s}^{2}+\rho_{s} \mathbf{T}-\frac{\rho_{s}}{\rho} \nabla p_{s}+\rho_{s} S \nabla T
$$

This allows to identify the tension $\mathbf{T}$ as

$$
\mathbf{T}=\left[\mathbf{v}_{\mathbf{i}} \times \vec{\omega}_{m i c r}\right]_{a v}=\kappa L<\mathbf{v}_{\mathbf{i}} \times \mathbf{s}^{\prime}>
$$

where we have denoted the average of $\mathbf{v}_{\mathbf{i}} \times \vec{\omega}_{\text {micr }}$, over the small vortex tangle contained in the mesoscopic volume $\Lambda$, with angular brackets, as in (3.7). Equation (3.12) makes explicit that the tension force $\mathbf{T}$ is related to the average curvature of vortex lines, as it was mentioned above.

Relation (3.12) may be rewritten in several equivalent forms, by taking into account (3.5) and some vectorial identities. For instance, $\left\langle\mathbf{s}^{\prime} \times \mathbf{v}_{\mathbf{i}}\right\rangle$ may be expressed, in the local induction approximation, as

$$
<\mathbf{s}^{\prime} \times \mathbf{v}_{\mathbf{i}}>=\tilde{\beta}<\mathbf{s}^{\prime} \times\left(\mathbf{s}^{\prime} \times \mathbf{s}^{\prime \prime}\right)>=-\tilde{\beta}<\mathbf{s}^{\prime \prime}>,
$$

where it has been taken into account that $\mathbf{s}^{\prime}$ is a unit vector.

Using (3.13), expression (3.12) may be rewritten as

$$
\mathbf{T}=\left[\mathbf{v}_{\mathbf{i}} \times \vec{\omega}_{m i c r}\right]_{a v}=\kappa L \tilde{\beta}<\left(\mathbf{s}^{\prime} \times \mathbf{s}^{\prime \prime}\right) \times \mathbf{s}^{\prime}>=\kappa L \tilde{\beta}<\mathbf{s}^{\prime \prime}>.
$$

Relation (3.14) is especially interesting because it explicitly describes the connection between $T$ and the curvature of the vortex lines, which is given by $\mathbf{s}^{\prime \prime}$. 
Another useful form for $\mathbf{T}$ may be obtained by using

$$
\mathbf{s}^{\prime \prime}=\left(\mathbf{s}^{\prime} \cdot \nabla\right) \mathbf{s}^{\prime}=-\mathbf{s}^{\prime} \times\left(\nabla \times \mathbf{s}^{\prime}\right)
$$

so that expression (3.13) may be rewritten as

$$
<\mathbf{s}^{\prime} \times \mathbf{v}_{\mathbf{i}}>=\tilde{\beta}<\mathbf{s}^{\prime} \times\left(\mathbf{s}^{\prime} \times \mathbf{s}^{\prime \prime}\right)>=\tilde{\beta}<\mathbf{s}^{\prime} \times\left(\nabla \times \mathbf{s}^{\prime}\right)>.
$$

Expressions (3.12), (3.14) and (3.16) will be used in the next sections.

\subsection{Limit of validity of the HVBK's equations}

As it can be seen by comparing (2.5) and (3.8), in expression (2.5) of the mutual friction force $\mathbf{F}_{n s}$ used in the HVBK equations the quantities $\left\langle\mathbf{s}^{\prime} \times\left(\mathbf{s}^{\prime} \times\left(\mathbf{v}_{n s}-\mathbf{v}_{\mathbf{i}}\right)\right)>\right.$ and $\left\langle\mathbf{s}^{\prime} \times\left(\mathbf{v}_{n s}-\mathbf{v}_{\mathbf{i}}\right)\right\rangle$ are approximated with $\hat{\omega} \times\left[\hat{\omega} \times\left(\mathbf{V}_{n s}-\tilde{\beta} \nabla \times \hat{\omega}\right)\right]$ and $\hat{\omega} \times\left(\mathbf{V}_{n s}-\tilde{\beta} \nabla \times \hat{\omega}\right)$ respectively, and $\left|\nabla \times \mathbf{v}_{s}\right|$ is approximated by $\kappa L$. The same approximations, when applied to the vortex tension $\mathbf{T}$, yield $(\tilde{\beta} \nabla \times \hat{\omega}) \times \vec{\omega}$.

We analyze in this section the limits of validity of the approximations leading to these expressions for $\mathbf{F}_{n s}$ and $\mathbf{T}$, and we will show that these limits are related to the neglect of the second order moments of the vector $\mathbf{s}^{\prime}$. We consider first the two averaged quantities $<\mathbf{s}^{\prime} \times\left(\mathbf{s}^{\prime} \times \mathbf{v}_{n s}\right)>$ and $\left\langle\mathbf{s}^{\prime} \times \mathbf{v}_{n s}>\right.$. We denote with $\mathbf{p}=<\mathbf{s}^{\prime}>$ and $\mathbf{V}_{n s}$ the averaged values of $\mathbf{s}^{\prime}$ and $\mathbf{v}_{n s}$, and with $\delta \mathbf{s}^{\prime}$ and $\delta \mathbf{v}_{n s}$ their respective fluctuations. The average value of the unit vector $\mathbf{s}^{\prime}$ is called tangle polarity [23]- 25], and - recall that the superfluid vorticity is quantized - is linked to the local averaged superfluid vorticity $\vec{\omega}$ by the relation

$$
\mathbf{p}=<\mathbf{s}^{\prime}>=\frac{1}{\Lambda L} \int \mathbf{s}^{\prime} d \xi=\frac{\vec{\omega}}{\kappa L}=\frac{\nabla \times \mathbf{V}_{s}}{\kappa L} .
$$

For a totally polarized tangle with all the tangents $\mathbf{s}^{\prime}$ parallel to each other, $|\mathbf{p}|=1$. In two dimensions, the polarization may be interpreted as $\left(n^{+}-n^{-}\right) /\left(n^{+}+n^{-}\right)$with $n^{+}$the vortices rotating in one direction and $n^{-}$the vortices rotating in opposite direction. Processes producing a partial separation of + and - vortices will thus change $\mathbf{p}$. This means that vortices may be partially agglomerated in such a way that + vortices will be predominant in some zones to the detriment of other ones, where - vortices will be. This leads to the formation of relatively large + and - vortices by local macroscopic accumulation of the corresponding + and - microscopic quantized vortices [7]. Thus, the dynamics of $\mathbf{p}$ may be certainly important and it can be influenced by the boundary conditions.

With the notation just introduced, neglecting the fluctuations of the counterflow velocity $\mathbf{V}_{n s}$, we may write $\mathbf{s}^{\prime}=\mathbf{p}+\delta \mathbf{s}^{\prime}$. Since $<\delta \mathbf{s}^{\prime}>=0$, we obtain

$$
\begin{aligned}
<\mathbf{s}^{\prime} \times\left(\mathbf{s}^{\prime} \times \mathbf{v}_{n s}\right)> & =\mathbf{p} \times\left(\mathbf{p} \times \mathbf{V}_{n s}\right)+<\delta \mathbf{s}^{\prime} \times\left(\delta \mathbf{s}^{\prime} \times \mathbf{V}_{n s}\right)>, \\
& <\mathbf{s}^{\prime} \times \mathbf{v}_{n s}>=\mathbf{p} \times \mathbf{V}_{n s} .
\end{aligned}
$$

Note that in the equation (2.5) only the first terms in the right-hand side of equations (3.18) and (3.19) appear. Indeed, recalling equation (3.17), the first terms in the right-hand side of equations (3.18) and (3.19) can be written as

$$
\mathbf{p} \times\left(\mathbf{p} \times \mathbf{V}_{n s}\right)=\frac{1}{\kappa L} \hat{\omega} \times\left[\vec{\omega} \times \mathbf{V}_{n s}\right]=<\mathbf{s}^{\prime} \times\left(\mathbf{s}^{\prime} \times \mathbf{v}_{n s}\right)>^{(H V B K)},
$$




$$
\mathbf{p} \times \mathbf{V}_{n s}=\frac{1}{\kappa L} \vec{\omega} \times \mathbf{V}_{n s}=<\mathbf{s}^{\prime} \times \mathbf{v}_{n s}>^{(H V B K)},
$$

whereas the other terms, quadratic in the fluctuations, have been neglected. In general, this will not be correct, for instance, in the limiting situations of an isotropic tangle, $\mathbf{p}=0$, but $<\mathbf{s}^{\prime} \times\left(\mathbf{s}^{\prime} \times \mathbf{v}_{n s}\right)>=(2 / 3) \mathbf{V}_{n s}[23]$.

Note also that in this simplified hypothesis, the second term in equation (3.18), dependent on the second moments of $\mathbf{s}^{\prime}$, can be neglected only if the orientational fluctuations of this unit vector in the small volume $\Lambda$ are very small, i.e. if most of the vortex lines in the volume have the same direction.

Furthermore, making use of equation (3.15), we obtain

$$
<\mathbf{v}_{\mathbf{i}}>=\tilde{\beta}<\mathbf{s}^{\prime} \times \mathbf{s}^{\prime \prime}>=\tilde{\beta}<\left(\nabla \times \mathbf{s}^{\prime}\right)-\left[\mathbf{s}^{\prime} \cdot\left(\nabla \times \mathbf{s}^{\prime}\right)\right] \mathbf{s}^{\prime}>=\tilde{\beta}<\left(\mathbf{U}-\mathbf{s}^{\prime} \mathbf{s}^{\prime}\right) \cdot\left(\nabla \times \mathbf{s}^{\prime}\right)>,
$$

with $\mathbf{U}$ the unit matrix and $\mathbf{s}^{\prime} \mathbf{s}^{\prime}$ the diadic product. Note that in the HVBK equations the quantity $\left\langle\mathbf{v}_{\mathbf{i}}>\right.$ is simply approximated by $\tilde{\beta}<\nabla \times \mathbf{s}^{\prime}>$ and therefore the quantity $<\left[\mathbf{s}^{\prime} \cdot\left(\nabla \times \mathbf{s}^{\prime}\right)\right] \mathbf{s}^{\prime}>$ is neglected.

From equations (3.20)-(3.21) it is seen that in the HVBK equations all the second-order moments of the fluctuations in $\mathbf{s}^{\prime}$ are neglected, that is $\mathbf{s}^{\prime} \simeq\left\langle\mathbf{s}^{\prime}\right\rangle=\mathbf{p}$, which implies $\left|<\mathbf{s}^{\prime}\right\rangle$ $\mid \simeq 1$ or

$$
\kappa L \simeq\left|\nabla \times \mathbf{V}_{s}\right|=|\vec{\omega}| .
$$

Last relation is the most critical hypothesis when it is used to evaluate the line density $L$. In fact, if in a mesoscopic region $\Lambda$ there are several vortex lines oriented in a random way, the line density $L$ in $\Lambda$ will be very different from $L \simeq\left|\nabla \times \mathbf{V}_{s}\right| / \kappa$ - which corresponds to an extreme polarization with all or almost all the lines pointing out in the same direction. We stress on this statement by a trivial example: let us consider in a small region $\Lambda$ a single vortex loop, then the average $\left|\left\langle\mathbf{s}^{\prime}\right\rangle\right|$ on this loop is zero, but this is not the case for the vortex line density $L$.

As a consequence, the HVBK equations describe correctly the interaction between the normal component and the vortex tangle only in mesoscopic regions with a high density array of vortex lines, all pointing in the same direction (totally polarized) and with the same curvature vector. This fact would explain the results of the numerical simulations obtained using the HVBK equations, which show the formation of macroscopic vortices and in which the microscopic chaotic dynamic behavior of the vortices does not appear. Of course, a confirmation of our statement should come from the results of numerical simulations of our equations and of the HVBK equations to problems with vortices not completely polarized.

\section{Generalization of HVBK equations}

In a previous paper [26] a hydrodynamical model of turbulent superfluids was formulated, which uses as fundamental fields the mass density $\rho$, the velocity $\mathbf{v}$ of the helium as a whole, the temperature $T$, the heat flux $\mathbf{q}$ and the line density $L$. In that work, only situations in which the tangle can be supposed approximately isotropic were considered, as is often made in the study of counterflow superfluid turbulence. In Ref. [23, the anisotropy of the vortex tangle was studied, restricting the study to homogeneous situations, and neglecting the influence of the vortex tension. In another paper [27] we have considered a more general situation, taking into account of inhomogeneities, anisotropy and polarization of the vortex tangle, studying the 
plane Couette and Poiseuille flow. For the sake of simplicity, in that work, we have neglected the vortex tension $\mathbf{T}$.

\subsection{New determination of $\mathbf{F}_{n s}$ and $\mathbf{T}$}

We want now to obtain expressions for $\mathbf{F}_{n s}$ and $\mathbf{T}$ overcoming some of the restrictions mentioned in the Section 3.2. Using the local-induction approximation, that is ignoring contribution on the self-induced velocity coming from the nonlocal portion of the vortex, and neglecting the fluctuations of the relative velocity $\mathbf{V}_{n s}$, from (3.8) we deduce that $\mathbf{F}_{n s}$ can be written [23]

$$
\mathbf{F}_{n s}=-\rho_{s} \kappa L\left[\alpha<\mathbf{U}-\mathbf{s}^{\prime} \mathbf{s}^{\prime}>+\alpha^{\prime}<\mathbf{W} \cdot \mathbf{s}^{\prime}>\right] \cdot \mathbf{V}_{n s}+\rho_{s} \kappa L \tilde{\beta}\left[\alpha<\mathbf{s}^{\prime} \times \mathbf{s}^{\prime \prime}>+\alpha^{\prime}<\mathbf{s}^{\prime \prime}>\right]
$$

with $\mathbf{W}$ the Ricci tensor (a completely antisymmetric third-order tensor such that $\mathbf{W} \cdot \mathbf{s}^{\prime} \cdot \mathbf{V}_{n s}=$ $\left.-\mathbf{s}^{\prime} \times \mathbf{V}_{n s}\right)$.

Introducing the tensor $\boldsymbol{\Pi}=\boldsymbol{\Pi}^{s}+\boldsymbol{\Pi}^{a}$, with $[23]$

$$
\mathbf{\Pi}^{s} \equiv \frac{3}{2}<\mathbf{U}-\mathbf{s}^{\prime} \mathbf{s}^{\prime}>, \quad \boldsymbol{\Pi}^{a} \equiv \frac{3}{2} \frac{\alpha^{\prime}}{\alpha}<\mathbf{W} \cdot \mathbf{s}^{\prime}>,
$$

the vectors $\mathbf{I}$ and $\mathbf{J}[17], 23]$

$$
\mathbf{I} \equiv \frac{\int \mathbf{s}^{\prime} \times \mathbf{s}^{\prime \prime} d \xi}{\int\left|\mathbf{s}^{\prime \prime}\right| d \xi}, \quad \mathbf{J} \equiv \frac{\int \mathbf{s}^{\prime \prime} d \xi}{\int\left|\mathbf{s}^{\prime \prime}\right| d \xi}
$$

and $c_{1} L^{1 / 2}=\frac{1}{\Lambda L} \int\left|\mathbf{s}^{\prime \prime}\right| d \xi$, a characteristic measure of the vortex tangle introduced by Schwarz [17, the mutual friction force (4.1) can be written in a compact way as

$$
\mathbf{F}_{n s}=\alpha \rho_{s} \kappa L\left[-\frac{2}{3} \boldsymbol{\Pi} \cdot \mathbf{V}_{n s}+\tilde{\beta} c_{1} L^{1 / 2}\left(\mathbf{I}+\frac{\alpha^{\prime}}{\alpha} \mathbf{J}\right)\right]
$$

The tensor $\boldsymbol{\Pi}$ is especially useful to describe the geometrical properties of the tangle related with the orientational distribution of the vortex lines, whose local direction is indicated by the unit tangent $\mathbf{s}^{\prime}$. When the tangle is not completely polarized nor fully isotropic, it should be described by a tensor, rather than by a vector, as it is usually done in the description of liquid crystals and of polymer solutions, where a tensor similar to $\Pi^{s}$ in (4.2) is used [28], [29]. The tensor $\boldsymbol{\Pi}$ does not contain information on the curvature of the lines because it does not contain $\mathbf{s}^{\prime \prime}$; this is furnished by the vectors $\mathbf{I}$ and $\mathbf{J}$.

The vortex tension $\mathbf{T}$ is also linked to the curvature vector $\mathbf{J}$; indeed it results

$$
\mathbf{T}=\left[\mathbf{v}_{\mathbf{i}} \times\left(\nabla \times \mathbf{v}_{s}\right)\right]_{a v}=\kappa L<\left(\tilde{\beta} \mathbf{s}^{\prime} \times \mathbf{s}^{\prime \prime}\right) \times \mathbf{s}^{\prime}>=\kappa L \tilde{\beta}<\mathbf{s}^{\prime \prime}>=\kappa L^{3 / 2} c_{1} \mathbf{J} .
$$

Observe that the tension of the vortex line, as the curvature vector, is zero in pure rotation with parallel straight vortex lines and in the isotropic tangle produced in well developed counterflow turbulence, but it is not so in the presence of simultaneous counterflow and rotation [30, 31] or in the first stages of the turbulence [33, 32] or in the transient states after sudden acceleration in plane Couette and Poiseuille flow. For instance, $\mathbf{T}$ could be different than zero in Kelvin helical vortex waves, which appear when a sufficiently intense counterflow is superposed to an axial rotation. In the transition from the array of straight lines to the array of helical vortex lines, a spiral tension would appear. The tension $\mathbf{T}$ would be a rotating quantity, as well as the curvature $\mathbf{s}^{\prime \prime}$ of the helical vortices. If the wavelength becomes shorter 
than the characteristic observational length, the average $\mathbf{T}$ will become equal to zero, but if the wavelength is high enough, it could lead to specific secondary flows of $\mathbf{v}_{s}$, through equation (2.4).

As a first modification to the HVBK equations we propose to take into account the fluctuations of the vector $\mathbf{s}^{\prime}$, which appear in the tensor $\left\langle\mathbf{U}-\mathbf{s}^{\prime} \mathbf{s}^{\prime}\right\rangle$, but, for sake of simplicity, we propose to neglect the fluctuations of $\mathbf{s}^{\prime \prime}$. This can be made following several way. Note indeed that in expressions (4.1) and (4.5) the quantity $\left\langle\mathbf{s}^{\prime \prime}\right\rangle$ appears, which is in general independent from $\left\langle\mathbf{s}^{\prime}\right\rangle=\mathbf{p}$ and from $\left\langle\mathbf{s}^{\prime} \mathbf{s}^{\prime}\right\rangle=\mathbf{U}-\boldsymbol{\Pi}$. However, our aim is to formulate an hydrodynamical model for a superfluid, with the lowest possible number of unknown quantities. As a consequence, we can try to find an approximate expression of $\left\langle\mathbf{s}^{\prime \prime}\right\rangle$ in terms of $\mathbf{p}$, $\nabla \mathbf{p}$ and $\mathbf{\Pi}$; the presence of $\nabla \mathbf{p}$ is logical because $\mathbf{s}^{\prime \prime}$ is related to the spatial derivative of $\mathbf{s}^{\prime}$; thus, although our expression will be only an approximate one, it makes clear that $\mathbf{p}$ and $\mathbf{\Pi}$ by themselves are not sufficient to give a description of $\mathbf{s}^{\prime \prime}$. This may be made, taking in mind relations (3.15). Indeed, putting $\left\langle\mathbf{s}^{\prime}\right\rangle=\mathbf{p}+\delta \mathbf{s}^{\prime}$, we have:

$$
<\mathbf{s}^{\prime \prime}>=-<\mathbf{s}^{\prime} \times\left(\nabla \times \mathbf{s}^{\prime}\right)>=-\mathbf{p} \times(\nabla \times \mathbf{p})-<\delta \mathbf{s}^{\prime} \times\left(\nabla \times \delta \mathbf{s}^{\prime}\right)>
$$

or also, noting that $\nabla \cdot\left(\mathbf{s}^{\prime} \mathbf{s}^{\prime}\right)=\left(\mathbf{s}^{\prime} \cdot \nabla\right) \mathbf{s}^{\prime}+\left(\nabla \cdot \mathbf{s}^{\prime}\right) \mathbf{s}^{\prime}$ :

$$
<\mathbf{s}^{\prime \prime}>=<\left(\mathbf{s}^{\prime} \cdot \nabla\right) \mathbf{s}^{\prime}>=<\nabla \cdot\left(\mathbf{s}^{\prime} \mathbf{s}^{\prime}\right)-\left(\nabla \cdot \mathbf{s}^{\prime}\right) \mathbf{s}^{\prime}>=-\nabla \mathbf{\Pi}-(\nabla \cdot \mathbf{p}) \mathbf{p}-<\left(\nabla \cdot \delta \mathbf{s}^{\prime}\right) \delta \mathbf{s}^{\prime}>
$$

As one sees, using the approximation

$$
<\mathbf{s}^{\prime \prime}>\simeq-\mathbf{p} \times(\nabla \times \mathbf{p})
$$

one neglect the quantity $<\delta \mathbf{s}^{\prime} \times\left(\nabla \times \delta \mathbf{s}^{\prime}\right)>$, i.e. an average of vectors orthogonal to $\delta \mathbf{s}^{\prime}$, while, if one puts

$$
<\mathbf{s}^{\prime \prime}>\simeq-\nabla \mathbf{\Pi}-(\nabla \cdot \mathbf{p}) \mathbf{p}
$$

one neglect the quantity $<\left(\nabla \cdot \delta \mathbf{s}^{\prime}\right) \delta \mathbf{s}^{\prime}>$, i.e. an average of vectors collinear to $\delta \mathbf{s}^{\prime}$.

The first approximation is true for very polarized or for completely non-polarized situations; in the first one, almost all tangent vectors to the lines go in the same direction, whereas in the last situation all of them go in different directions, in such a way that they cancel each other and yield null polarization. The second approximation is more useful in intermediate situations. Another possibility, which may be useful in situations with a high but not total polarization, would be to use an average of the two previous approximated expressions (4.8) and (4.9), but we will not deal with it for the sake of simplicity. In the following we will use for $\left\langle\mathbf{s}^{\prime \prime}\right\rangle$ the approximation (4.8).

To what concerns the vectors $\mathbf{I}$ and $\mathbf{J}$, and the vortex tension $\mathbf{T}$, with these approximations in mind, we have the following constitutive relations

$$
\begin{gathered}
c_{1} L^{1 / 2} \mathbf{I}=<\mathbf{s}^{\prime} \times \mathbf{s}^{\prime \prime}>\simeq<\mathbf{U}-\mathbf{s}^{\prime} \mathbf{s}^{\prime}>\cdot \nabla \times \mathbf{p}, \\
c_{1} L^{1 / 2} \mathbf{J}=<\mathbf{s}^{\prime \prime}>\simeq-\mathbf{p} \times(\nabla \times \mathbf{p})=(\mathbf{p} \cdot \nabla) \mathbf{p}-\frac{1}{2} \nabla \mathbf{p}^{2} .
\end{gathered}
$$

The required equations for $\mathbf{F}_{n s}$ and $\mathbf{T}$ are

$$
\begin{aligned}
\mathbf{F}_{n s}=-\rho_{s} \kappa L \alpha<\mathbf{U}-\mathbf{s}^{\prime} \mathbf{s}^{\prime} & >\cdot\left[\mathbf{V}_{n s}-\tilde{\beta}(\nabla \times \mathbf{p})\right]+\rho_{s} \kappa L \alpha^{\prime}\left[\mathbf{p} \times \mathbf{V}_{n s}-\tilde{\beta} \mathbf{p} \times(\nabla \times \mathbf{p})\right]= \\
& =-\frac{2}{3} \rho_{s} \kappa L \alpha \boldsymbol{\Pi} \cdot\left[\mathbf{V}_{n s}-\tilde{\beta}(\nabla \times \mathbf{p})\right],
\end{aligned}
$$




$$
\rho_{s} \mathbf{T}=-\rho_{s} \kappa L \tilde{\beta} \mathbf{p} \times(\nabla \times \mathbf{p}) .
$$

In Section 5, some illustration of (4.12) and (4.13) will be presented, with different form of the field $\Pi$. But, to complete our hydrodynamical model, we must first add to equations (4.12) -(4.13) an evolution equation for the line density $L$.

\subsection{Generalized Vinen equation including polarization, anisotropy, and in- homogeneities}

In expressions (4.1) and (4.5) - or (4.12) and (4.13) - for $\mathbf{F}_{n s}$ and $\mathbf{T}$, it appears $L$; therefore, to have a full description for the evolution of the system, an evolution equation for $L$ is needed.

The evolution equation for $L$ in counterflow superfluid turbulence was formulated by Vinen [32. Assuming homogeneous turbulence, such an equation is

$$
\frac{d L}{d t}=\alpha_{v} V_{n s} L^{3 / 2}-\beta_{v} \kappa L^{2}
$$

with $\alpha_{v}$ and $\beta_{v}$ dimensionless parameters.

A microscopic derivation of this equation was given by Schwarz [15, 17] on the basis of the dynamics of the vortices, neglecting however a term in the average curvature vector $\mathbf{s}^{\prime \prime}$. In Ref. [34], because we were interested to study wall effects on the evolution of $L$, the following extension of equation (4.14) was written

$$
\frac{d L}{d t} \simeq \alpha c_{1} \mathbf{V}_{n s} \cdot \mathbf{I} L^{3 / 2}+\alpha^{\prime} c_{1} \mathbf{V}_{n s} \cdot \mathbf{J} L^{3 / 2}-\alpha \tilde{\beta} c_{2} L^{2},
$$

with $c_{1}$, I and $\mathbf{J}$ defined in equations (4.3), $c_{1}$ in the line below (4.3), and $c_{2}=\frac{1}{\Lambda L^{2}} \int\left|\mathbf{s}^{\prime \prime}\right|^{2} d \xi$.

Substituting in this equation the relations (4.104.11), the following evolution equation for $L$ which takes into account the polarization and the anisotropy of the tangle is obtained

$$
\frac{d L}{d t} \simeq \alpha L \mathbf{V}_{n s} \cdot\left\langle\mathbf{U}-\mathbf{s}^{\prime} \mathbf{s}^{\prime}>\cdot(\nabla \times \mathbf{p})-\alpha^{\prime} L \mathbf{V}_{n s} \cdot \mathbf{p} \times(\nabla \times \mathbf{p})-\alpha \tilde{\beta} c_{2}(\mathbf{p}) L^{2}\right.
$$

In Refs. [27], 31]-33], [35]-37] Vinen's equation was modified to describe more complex situations, as for instance the coupled situation of counterflow and rotation and in Couette and Poiseuille flows. Taking in mind the equation (4.16) and the proposed equation of Ref. [27], the previous equation would become

$\frac{d L}{d t} \simeq \alpha L \mathbf{V}_{n s} \cdot\left\langle\mathbf{U}-\mathbf{s}^{\prime} \mathbf{s}^{\prime}>\cdot(\nabla \times \mathbf{p})-\alpha^{\prime} L \mathbf{V}_{n s} \cdot \mathbf{p} \times(\nabla \times \mathbf{p})-\alpha \tilde{\beta} \kappa L^{2}[1-\sqrt{|\mathbf{p}|}][1-B \sqrt{|\mathbf{p}|}]\right.$,

where $B$ is a dimensionless coefficient lower than 1. A rigorous derivation of its form would require a microscopic extension of Schwarz's model including rotation effects. To check the consistency of the obtained evolution equation (4.17) for the vortex line density equation, in the next subsection an analysis based on the formalism of linear irreversible thermodynamics will be made.

When inhomogeneities in the line density $L$ are taken into account, the evolution equation for line density $L$ must include a vortex density flux $\mathbf{J}^{L}$, as [26]

$$
\frac{\partial L}{\partial t}+\nabla \cdot \mathbf{J}^{L}=\sigma^{L},
$$


where $\sigma_{L}$ stands for the right-hand side of equation (4.17). The flux $\mathbf{J}^{L}$ can also be expressed in terms of a convective part $L \mathbf{v}^{L}$ with $\mathbf{v}^{L}$ the tangle velocity and a dissipative part $\mathbf{J}_{d}^{L}$. The particular form of $\mathbf{J}^{L}$ is also open to the debate. One can suppose that $\mathbf{J}^{L}$ is an independent variable [38], or, more simply, supposing that $\mathbf{J}^{L}$ is a dependent field: we have found [26] for it the form $\mathbf{J}^{L}=\nu_{0} \mathbf{q}, \nu_{0}$ being a coefficient describing the interaction between the vortex tangle and the heat flux $\mathbf{q}$, which is linked to the counterflow velocity by the relation $\mathbf{q}=\rho_{s} T S \mathbf{V}_{n s}$. The heat flux q may also be expressed in terms of $\nabla T$ and $\nabla L$ as [26]

$$
\mathbf{q}=-\frac{\eta}{\kappa L_{0}} \nabla T-\frac{\chi_{0}}{\kappa L_{0}} \nabla L
$$

Thus, the dissipative part of the vortex flux $\mathbf{J}_{d}^{L}$ may be expressed as

$$
\mathbf{J}_{d}^{L}=-\frac{\nu_{0} \eta_{0}}{\kappa L_{0}} \nabla T-\frac{\nu_{0} \chi_{0}}{\kappa L_{0}} \nabla L .
$$

In isothermal situations, $\mathbf{J}_{d}^{L}$ may be written as $\mathbf{J}_{d}^{L}=-D \nabla L$, with $D$ a vortex diffusion coefficient defined by $D=\nu_{0} \chi_{0} / \kappa L_{0}[34]$.

\subsection{Consequences of Onsager-Casimir reciprocity relation}

In this Subsection, we show that another modification of the vortex line density evolution equation is necessary to insure the thermodynamic consistency of the evolution equations for $L$ and for $\mathbf{V}_{s}$, according to the formalism of linear irreversible thermodynamics [37, 39, 40]. This analysis requires the presence of another term linked to the tension of vortices.

We follow the general lines of [37, 39, 40] with the aim to study the consequences of the Onsager-Casimir reciprocity relations on the evolution equations of $\mathbf{V}_{s}$ and $L$ proposed in the previous Sections. The Onsager relations were derived at the microscopic scale by using fluctuation theory and microscopic time reversibility, and they do not depend on the details of the microscopic models; this is the reason that their validity is not restricted to dilute gases, but it has been checked for different kinds of systems (dilute and dense gases, liquids, solids, multicomponent fluids, artificial and biological membranes).

According to the formalism of nonequilibrium thermodynamics one may obtain evolution equations for $\mathbf{V}_{s}$ and $L$ by writing $d \mathbf{V}_{s} / d t$ and $d L / d t$ in terms of their conjugate thermodynamic forces $-\rho_{s} \mathbf{V}_{n s}$ and $\epsilon_{V}$. The evolution equation for $\mathbf{V}_{s}$, neglecting inhomogeneous contributions of pressure, temperature and velocity, in an inertial frame, is written

$$
\rho_{s} \frac{d \mathbf{V}_{s}}{d t}=-\mathbf{F}_{n s}-\rho_{s} \mathbf{T}=\alpha \rho_{s} \kappa L \frac{2}{3} \boldsymbol{\Pi} \cdot \mathbf{V}_{n s}-\alpha \rho_{s} \kappa L \tilde{\beta} \frac{2}{3} \boldsymbol{\Pi} \cdot(\nabla \times \mathbf{p})+\rho_{s} \kappa L \tilde{\beta} \mathbf{p} \times(\nabla \times \mathbf{p})
$$

and the evolution equation for $L$ is written

$$
\frac{d L}{d t}=\alpha L \mathbf{V}_{n s} \cdot\left\langle\mathbf{U}-\mathbf{s}^{\prime} \mathbf{s}^{\prime}>\cdot(\nabla \times \mathbf{p})-\alpha^{\prime} L \mathbf{V}_{n s} \cdot \mathbf{p} \times(\nabla \times \mathbf{p})-\alpha \tilde{\beta} c_{2}(\mathbf{p}) L^{2}\right.
$$

However, in the right-hand side of (4.22) additional contributions must be included to make (4.22) thermodynamically consistent with (4.21).

Similarly to that presented in [37], we write $d \mathbf{V}_{s} / d t$ and $d L / d t$ in matrix form using the equations (4.21) and (4.22), and by means of Onsager-Casimir reciprocity we obtain an additional contribution to the evolution equation for $L$. The result is

$$
\left(\begin{array}{l}
\frac{d \mathbf{V}_{s}}{d t} \\
\frac{d L}{d t}
\end{array}\right)=L\left(\begin{array}{cc}
-\frac{2}{3} \frac{\alpha \kappa}{\rho_{s}} \boldsymbol{\Pi} & -\frac{2}{3} \frac{\alpha}{\rho_{s}}(\nabla \times \mathbf{p}) \cdot \boldsymbol{\Pi}+\frac{1}{\rho_{s}} \mathbf{p} \times(\nabla \times \mathbf{p}) \\
-\frac{\alpha}{\rho_{s}} \frac{2}{3}(\nabla \times \mathbf{p}) \cdot \boldsymbol{\Pi}+\frac{1}{\rho_{s}} \mathbf{p} \times(\nabla \times \mathbf{p}) & -\frac{\alpha \tilde{\beta}}{\epsilon_{V}} L c_{2}(\mathbf{p})
\end{array}\right)\left(\begin{array}{c}
-\rho_{s} \mathbf{V} \\
\epsilon_{V}
\end{array}\right)
$$


where for $c_{2}(\mathbf{p})$ we can choose the expression $c_{2}(\mathbf{p})=(1-\sqrt{|\mathbf{p}|})(1-B \sqrt{|\mathbf{p}|})$ found in Ref. [27.

Therefore the equation for $d L / d t$ becomes

$$
\frac{d L}{d t}=\alpha L \mathbf{V}_{n s} \cdot<\mathbf{U}-\mathbf{s}^{\prime} \mathbf{s}^{\prime}>\cdot(\nabla \times \mathbf{p})-\left(1+\alpha^{\prime}\right) L \mathbf{V}_{n s} \cdot \mathbf{p} \times(\nabla \times \mathbf{p})-\alpha \tilde{\beta} c_{2}(\mathbf{p}) L^{2},
$$

or, similarly,

$$
\frac{d L}{d t}=\frac{2}{3} \alpha L \mathbf{V}_{n s} \cdot \boldsymbol{\Pi} \cdot(\nabla \times \mathbf{p})-\frac{1}{\kappa \tilde{\beta}} \mathbf{V}_{n s} \cdot \mathbf{T}-\alpha \tilde{\beta}(1-\sqrt{|\mathbf{p}|})(1-B \sqrt{|\mathbf{p}|}) L^{2}
$$

The new term not contained in the evolution equation (4.22) for $L$ is the coupling term between $d L / d t$ and $-\rho_{s} \mathbf{V}_{n s}$ in the matrix in (4.23), which is linked to the tension of vortices and it is null when the tension $\mathbf{T}$ is null.

Note that, introducing the tensor

$$
\mathbf{\Pi}^{\prime}=<\mathbf{U}-\mathbf{s}^{\prime} \mathbf{s}^{\prime}>+\frac{1+\alpha^{\prime}}{\alpha}<\mathbf{W} \cdot \mathbf{s}^{\prime}>
$$

the system (4.23) can be written

$$
\left(\begin{array}{l}
\frac{d \mathbf{v}_{s}}{d t} \\
\frac{d L}{d t}
\end{array}\right)=L\left(\begin{array}{cc}
-\frac{2}{3} \frac{\alpha \kappa}{\rho_{s}} \boldsymbol{\Pi} & -\frac{2}{3} \frac{\alpha}{\rho_{s}} \boldsymbol{\Pi}^{\prime} \cdot(\nabla \times \mathbf{p}) \\
-\frac{2}{3} \frac{\alpha}{\rho_{s}} \boldsymbol{\Pi}^{\prime} \cdot(\nabla \times \mathbf{p}) & -\frac{\alpha \tilde{\beta}}{\epsilon_{V}} L c_{2}(\mathbf{p})
\end{array}\right)\left(\begin{array}{c}
-\rho_{s} \mathbf{V} \\
\epsilon_{V}
\end{array}\right)
$$

\section{Application to rotating counterflow turbulence}

Combination of counterflow and rotation is especially interesting in the context of the present paper, because it provides intermediate situations between an isotropic tangle and a totally anisotropic array of parallel vortices. The polarization and anisotropy of the tangle depend on the ordering influence of the rotation, which tend to align the vortices parallel to the rotation axis, and the randomizing aspects of the counterflow. In this section we will apply the previous results.

\subsection{Pure counterflow}

Experimental observations [18] and numerical simulations [17] in counterflow superfluid turbulence show anisotropy in the vortex line distribution with vortices concentrated in planes orthogonal to $\mathbf{V}_{n s}$. Assuming $\mathbf{V}_{n s}$ in the direction of the $x$ axis and isotropy in planes orthogonal to it, one can choose for the tensor $\boldsymbol{\Pi}$ (equation (4.2)) the following expression [23]

$$
\boldsymbol{\Pi}_{H}=\boldsymbol{\Pi}_{H}^{s}=\frac{3}{2}\left(\begin{array}{ccc}
2 a & 0 & 0 \\
0 & 1-a & 0 \\
0 & 0 & 1-a
\end{array}\right)
$$

here $a$ is the anisotropic parameter linked to the coefficients $I_{\|}$and $I_{\perp}$ introduced by Schwarz [17] by the relations $I_{\|}=2 a$ and $I_{\perp}=1-a$. Being known the values of coefficient $a$, by accurate measurements of second sound, the second order moments of the unit vector $\mathbf{s}^{\prime}$ remain determined. Indeed, it results

$$
<s_{x}^{\prime 2}>=1-2 a, \quad<s_{y}^{\prime 2}>=<s_{z}^{\prime 2}>=a .
$$


For the vector I, we propose, according to (4.10), the following constitutive relation

$$
c_{1} L^{1 / 2} \mathbf{I}=<\mathbf{s}^{\prime} \times \mathbf{s}^{\prime \prime}>\simeq<\mathbf{U}-\mathbf{s}^{\prime} \mathbf{s}^{\prime}>\cdot \nabla \times \mathbf{p},
$$

The asymmetric part of the tensor $\mathbf{\Pi}$, the curvature vector $\mathbf{J}$ and the vortex tension $\mathbf{T}$ in this case are zero, owing to the supposed isotropy of the tangle in planes orthogonal to $\mathbf{V}_{n s}$, which implies $\left\langle\mathbf{s}^{\prime \prime}\right\rangle=0$. Introducing (5.1) into (4.12) and (4.17) we could obtain the influence of the anisotropy $a$ on the mutual friction force $\mathbf{F}_{n s}$ and the evolution of $L$.

\subsection{Simultaneous counterflow and rotation}

- Under the simultaneous influence of counterflow velocity $\mathbf{V}_{n s}$ and rotation with angular speed $\boldsymbol{\Omega}$, rotation tends to align vortex lines parallel to rotation axis, whereas counterflow velocity tends to produce a disordered tangle. In these situations one has partially polarized tangles, requiring the full detailed analysis presented here. We assume that the total ensemble of vortex lines is a superposition of both contributions [23]

$$
\boldsymbol{\Pi}^{s}=(1-b) \boldsymbol{\Pi}_{H}^{s}+b \boldsymbol{\Pi}_{R}^{s}, \quad \boldsymbol{\Pi}^{a}=c \boldsymbol{\Pi}_{R}^{a} .
$$

In (5.4), $b$ and $c$ are parameters between 0 and 1, which depend on $\boldsymbol{\Omega}$ and $\mathbf{V}_{n s}$, describing the relative weight of the array of vortex lines parallel to $\boldsymbol{\Omega}$ and the disordered tangle of counterflow.

In Ref. 23], we examined explicitly two simplified situations: $\mathbf{V}$ parallel to $\boldsymbol{\Omega}$ and $\mathbf{V}$ orthogonal to $\boldsymbol{\Omega}$; in the first case there is cylindrical symmetry with respect to the rotation axis; in the second one, no symmetries are present in the vortex tangle.

In the case $\mathbf{V} \| \boldsymbol{\Omega}$, choosing $\mathbf{V}$ and $\boldsymbol{\Omega}$ in the direction of the $x$ axis, one has for the symmetric part of tensor $\Pi$ :

$$
\boldsymbol{\Pi}^{s}=\Pi_{H}^{s}+\Pi_{R}^{s}=\frac{3}{2}(1-b)\left(\begin{array}{ccc}
2 a & 0 & 0 \\
0 & 1-a & 0 \\
0 & 0 & 1-a
\end{array}\right)+\frac{3}{2} b\left(\begin{array}{ccc}
0 & 0 & 0 \\
0 & 1 & 0 \\
0 & 0 & 1
\end{array}\right)
$$

which can be written:

$$
\boldsymbol{\Pi}^{s}=\frac{3}{2}<\mathbf{U}-\mathbf{s}^{\prime} \mathbf{s}^{\prime}>=\frac{3}{2}\left(\begin{array}{ccc}
2 a(1-b) & 0 & 0 \\
0 & 1-a(1-b) & 0 \\
0 & 0 & 1-a(1-b)
\end{array}\right) .
$$

Therefore, we have $\left\langle{s_{x}^{\prime}}^{2}\right\rangle=1-2 a(1-b),\left\langle s_{y}^{\prime 2}\right\rangle=<s_{z}^{\prime 2}>=a(1-b)$, and $I_{\|}=2 a(1-b)$, $I_{\perp}=1-a(1-b)$. Once known the coefficient $a$, from experiments in pure counterflow, from (5.6) we can obtain the dependence of $b$ from the second moments of $\mathbf{s}^{\prime}$.

In the second situation, when the counterflow velocity $\mathbf{V}$ is orthogonal to the angular velocity $\Omega$, choosing $\Omega$ in the direction of $x$ axis and $\mathbf{V}$ in the one of the $z$ axis, one has, for $\Pi^{s}$ :

$$
\boldsymbol{\Pi}^{s}=(1-b) \boldsymbol{\Pi}_{H}^{s}+b \boldsymbol{\Pi}_{R}^{s}=\frac{3}{2}\left(\begin{array}{ccc}
(1-a)(1-b) & 0 & 0 \\
0 & (1-a)(1-b)+b & 0 \\
0 & 0 & 2 a(1-b)+b
\end{array}\right) .
$$

As a consequence, it results

$$
<s_{x}^{\prime 2}>=a(1-b)+b, \quad<s_{y}^{\prime 2}>=a(1-b), \quad<s_{z}^{2}>=(1-2 a)(1-b) .
$$


The antisymmetric part of tensor $\boldsymbol{\Pi}$. which depends only on $\boldsymbol{\Pi}_{R}$, furnishes the polarity of the tangle; in fact, being $\mathbf{W}$ a constant tensor, one can write:

$$
\boldsymbol{\Pi}^{a}=\frac{3}{2} \frac{\alpha^{\prime}}{\alpha} c \mathbf{W} \cdot \hat{\mathbf{\Omega}}=\frac{3}{2} \frac{\alpha^{\prime}}{\alpha} c\left(\begin{array}{ccc}
0 & 0 & 0 \\
0 & 0 & 1 \\
0 & -1 & 0
\end{array}\right),
$$

from which we deduce $\left.\left.\left\langle s_{x}^{\prime}\right\rangle=\frac{\alpha^{\prime}}{\alpha} c,<s_{y}^{\prime}\right\rangle=<s_{z}^{\prime}\right\rangle=0$.

A microscopic evaluation of coefficients $b$ and $c$ was made in Ref. 23], based on a paramagnetic analogy, which reflects the competition between the orienting effects of $\boldsymbol{\Omega}$ and the randomizing effects of $\mathbf{V}_{n s}$, respectively analogous to the orienting effects of a magnetic field $\mathbf{H}$ on magnetic dipoles $\mu$ and the randomizing effects of thermal excitations. In rotating counterflow, the rotation $\Omega$ orients the vortices along its direction, in an analogous way to $\mathbf{H}$, whereas the counterflow $\mathbf{V}_{n s}$ plays a disordering role. We found, using the Langevin model of paramagnetism,

$$
<s_{x}^{\prime}>=\operatorname{coth} x-\frac{1}{x}
$$

and

$$
<s_{x}^{\prime 2}>=1+\frac{2}{x}\left[\frac{1}{x}-\operatorname{coth} x\right],
$$

with $x$ proportional to $\Omega \kappa / V_{n s}^{2}$. Similar situations would be found in Couette and Poiseuille flow where the velocity gradient, instead that a rotation, contributes to orient the vortices [41, 42] with $\Omega$ replaced by the local shear rate.

A particular illustration of the combination of (5.4) and (4.12) could be provided by the decay of small perturbations of $\mathbf{V}_{n}-\mathbf{V}_{s}$. According to (2.3) and (2.4) and the assumption $\mathbf{T}=\mathbf{0}$ and $\nabla T \cong 0$, one would have, in a linear approach

$$
\frac{\partial\left(\mathbf{V}_{n}-\mathbf{V}_{s}\right)}{\partial t}=-\frac{2}{3} \alpha \frac{\rho}{\rho_{n}} \kappa L(1-b)\left(\mathbf{V}_{n}-\mathbf{V}_{s}\right)+\frac{\eta}{\rho_{n}} \nabla^{2} \mathbf{V}_{n}
$$

being $\mathbf{V}_{n}-\mathbf{V}_{s}$ along $x$ axis. The first term on the right hand side, describes $\frac{\rho}{\rho_{s} \rho_{n}} \mathbf{F}_{n s}$, taking into account (4.12) and (5.4).

For long-wave perturbations, $\nabla^{2} \mathbf{V}_{n}$ will be small as compared to the first term and the decay of $\mathbf{V}_{n}-\mathbf{V}_{s}$ to its steady state value will be exponential, with a relaxation time depending on the anisotropy parameter $b$. This time could provide a measurement of $b$ independent of the measurement provided by the attenuation of the second sound along different axis of the system. An analogous analysis could be carried out to explore the anisotropy parameter $a$ introduced in (5.1) for tangles in pure counterflow.

A second particular illustration may underline the role of the term in $\mathbf{I}$ in expression (4.4) for $\mathbf{F}_{n s}$. This would be reflected in the temperature gradient needed to maintain a steady state value of $\mathbf{V}_{n s}$, at constant pressure. Under these conditions, one has

$$
-2 \rho_{s} S \nabla T+2 \mathbf{F}_{n s}+\eta \nabla^{2} \mathbf{V}_{n}=0 .
$$

Thus, neglecting, for simplicity, the term in $\eta \nabla^{2} \mathbf{V}_{n}$ and using (4.4) for $\mathbf{F}_{n s}$, we have

$$
\alpha \kappa L\left[\frac{2}{3} \boldsymbol{\Pi} \cdot \mathbf{V}_{n s}+\tilde{\beta} c_{1} L^{1 / 2} \mathbf{I}\right]=S \nabla T .
$$

It is clear that the presence of $\mathbf{I}$ modifies the relation between $\mathbf{V}_{n s}$ and $\nabla T$; this could allow to measure the influence of the corresponding term.

These two simple illustrations show that the generalized equation for $\mathbf{F}_{n s}$ considered in (4.4) is indeed expected to have specific applications. 


\section{Conclusions}

Summarizing, in this work we propose to substitute in the HVBK equations the expression of the mutual friction force $\mathbf{F}_{n s}$ (equation (2.5)) with the equation (4.12), to take into account of the second-order moment of $\mathbf{s}^{\prime}$, allowing in this way that not all the vortex lines in the small volume element under consideration have the same direction. Analogously, the expression (2.7) for the tension $\mathbf{T}$ has been replaced by equation (4.13). The coefficients $b$ and $c$ appearing in (5.4) can be related to the counterflow velocity and to the angular velocity by the relations (5.8), with $\left\langle s_{x}^{\prime}\right\rangle$ and $\left\langle s_{x}^{\prime 2}\right\rangle$ expressed by (5.10) and (5.11). For the vectors $\mathbf{I}$ and $\mathbf{J}$ we have chosen the constitutive relations (4.10) and (4.11).

Another important modification consists in adding to the evolution equations for $\mathbf{V}_{n}$ and $\mathbf{V}_{s}$ an evolution equation for $L$ (see eq. (4.17), or (4.25) and (4.27)), including the effects of polarization anisotropy and inhomogeneities. In fact, in a general situation, it is not correct in (3.23) to substitute $\kappa L$ with the modulus of the curl of $\mathbf{V}_{s}$. But, in a first approximation it should be valid when a relevant polarization is present in the vortices, that is when the polarity vector $\mathbf{p}$ approximate to 1 . So, the results obtained by some authors which measure the decay of $L$ or compute a fluid viscosity by means of this relationship are good when the polarization of the vortex tangle is high enough. Relations (4.17) and (4.18) avoid this simplification.

Our approach is not an exact description of superlfuid hydrodynamics, but an approximation which, in the mentioned situations, is more satisfactory that the HVBK assumption. In fact, an exact description of the hydrodynamics would require not only to analyze $\mathbf{s}^{\prime}$ but also $\mathbf{s}^{\prime \prime}, \mathbf{s}^{\prime \prime \prime}$ and so on, and to deal not only with their respective average values, but also with their second and higher-order moments as well as with their correlations. Something similar happens in classical turbulence, in whose detailed description a hierarchy of equations for the second and higher-order models of the fluctuations velocity appears, and is usually truncated by some approximate arguments. Here, instead of the moments of the random component of the fluctuating velocity, the higher-order moments of $\mathbf{s}^{\prime}, \mathbf{s}^{\prime \prime}, \mathbf{s}^{\prime \prime \prime}$ and so on should appear, but this would be not practical at all, and conveniently truncated approaches must be analyzed.

The domain of validity of the new equations does not depend only on $L$ but also on polarization, which may have different values for a same value of $L$; for instance, in rotating counterflow, a same $L$ may have different polarizations depending on the value of the angular velocity; analogously, as mentioned above, towing parallel thin cylinders or towing a grid of two orthogonal families of parallel cylinders, but at different speeds, could give a same $L$ but with polarization values different from each other; finally, for short values of $L$, and not very developed turbulence, pinned vortices may contribute to the polarization, and could provide situations for an analysis.

A possible experiment of interest would be to compare the turbulence produced by towing through the superfluid a set of very thin cylinders parallel to each other, and that produced by towing a grid composed of two orthogonal families of parallel thin cylinders, perpendicular to each other. In the first case, a strong polarization parallel to the cylinders is expected, whereas for the grid much less polarization should be expected. A fourth possible check would be, as mentioned in Section 4.1, the influence of the tension $T$ in the dynamics of long wavelength helical vortex lines.

In Section 5, we have concentrated our attention on rotating counterflow; another illustration of the possible physical consequences of the tension $\mathbf{T}$, may be found in steady Poiseuille flows in an isothermal situation. There, addition of (2.3) and (2.4) leads for the equation 
describing the velocity profile of the normal component

$$
-\nabla p+\eta \nabla^{2} \mathbf{V}_{n}+\rho_{s} \mathbf{T}=\mathbf{0} .
$$

Thus, if $\mathbf{T}=\mathbf{0}$, the $\mathbf{V}_{n}$ profile will have the typical parabolic form of Newtonian fluid. Modifications of fluid velocity profile have been studied by Godfrey and Barenghi [42]. From (6.1), these modifications would be related to the form of T. A completely polarized array of parallel rectilinear vortices or a completely isotropic vortex tangle would have $\mathbf{T}=\mathbf{0}$ and would not modify the form of the $\mathbf{V}_{n}$ profile. However, net tension different from zero could arise at relatively low fluxes, due to the influence of vortices pinned to the walls, which in the presence of the flow would have a curvature opposite to the velocity of the flow. In particular, they would have an influence on a small-amplitude oscillating flow along a cylinder with pinned vortices. An analysis of such situation would provide another possible check of the equations proposed here. A third possible check would be to study the decay of counterflow rotating turbulence for different values of the angular speed, as it has been mentioned in Section 5 .

Finally, to mention yet another situation where the effects of the polarization are important, and for which experimental data have been recently available, is the eddy formation in two-dimensional counterflow in the presence of a transverse cylinder, in which two macroscopic vortices appear in the upstream part of the cylinder [7, in contrast with the usual vortices appearing downstream of the cylinder in plane flows of normal viscous fluids. These macroscopic vortices, rotating in opposite directions, could arise from a separation of clockwise and counterclockwise microscopic vortices induced by the presence of the cylinder. Though this is, at present, only a plausible guess, but not a definite explanation of this interesting phenomenon, it allows to see, at least tentatively, a situation in which the results of this work would be of special interest. Indeed, far upstream from the cylinder, the flow would have vortices (i.e. $L$ different from 0), but null polarity. Near the cylinder, in the macroscopic vortex regions, the microscopic vortices would be thorouhgly polarized, in two opposite directions. In between, there would be a process in which polarization would raise from 0 to 1 . In the corresponding process, equation (2.4) for $\mathbf{F}_{n s}$ cannot be used, because it does not account for any change of polarization. Instead, in (4.4) the polarization is present through the tensor $\Pi$. Explaining in full detail the results of Ref [7] seems not straightforward, but it seems a good field for application of the generalized equations proposed in this paper.

\section{Acknowledgments}

We acknowledge the support of the Acción Integrada España-Italia (Grant S2800082F HI20040316 of the Spanish Ministry of Science and Technology and grant IT2253 of the Italian MIUR). DJ acknowledges the financial support from the Dirección General de Investigación of the Spanish Ministry of Education under grant FIS 2009-13370-C02-01 and of the Direcció General de Recerca of the Generalitat of Catalonia, under grant 2009 SGR-00164. MSM and MS acknowledge the financial support by "Fondi 60\%" of the University of Palermo. MS acknowledges the "Assegno di ricerca: Studio della turbolenza superfluida e della sua evoluzione" of the University of Palermo. 


\section{References}

[1] Donnelly, R.J. Quantized vortices in helium II. Cambridge: Cambridge University Press, 1991

[2] Nemirovskii, S.K., Fiszdon W., Chaotic quantized vortices and hydrodynamic processes in superfluid helium Rev. Mod. Phys. 67, 37-84 (1995)

[3] Barenghi, C.F., Donnelly, R.J., Vinen, W.F., Quantized Vortex Dynamics and Superfluid Turbulence Berlin: Springer, 2001

[4] Vinen, W.F., Niemela, J., Quantum Turbulence J. Low Temp. Phys. 128, 167-231 (2002)

[5] Hall, H.E., Vinen, W.F., The rotation of liquid helium II. The theory of mutual friction in uniformly rotating helium II Proc. Roy. Soc. A238, 204-214 (1956)

[6] Bekarevich, I.L., Khalatnikov, I.M., Phenomenological derivation of the equation of vortex motion in He II Sov.Phys. JETP 13, 643-646 (1961)

[7] Zhang, T., Van Sciver, S.W., Large-scale turbulent flow around a cylinder in counterflow superfluid ${ }^{4}$ He Nature Phys. 1, 36-38 (2005) DOI 10.1038/nphys114

[8] Martin, K.P., Tough, J.T. Evolution of superfluid turbulence in thermal counterflow Phys. Rev. B 27, 2788-2799 (1983)

[9] Mongiovì, M.S., Jou, D. Generalization of Vinen's equation including transition to superfluid turbulence, J. Phys. Condensed Matter 17, 4423-4440 (2005)

[10] Swanson, C.J., Donnelly, R.J., Instability of Taylor-Couette fluid in Helium II Phys. Rev. Letters 67, 1578-1581 (1991)

[11] Barenghi, C. F., Vortices and the Couette fluid of helium II Phys. Rev. B 45, 2290-2293 (1992)

[12] Henderson, K.L., Barenghi, C.F., End effects in rotating helium II Physica B 184-288, 67-68 (2000)

[13] Holm, D.D., Renormalized HVBK dynamics for superfluid helium turbulence In: Barenghi, C.F.,. Donnelly, R.J, Vinen, W.F. (eds.) Quantized Vortex Dynamics and Superfluid Turbulence Proceedings 2000. Berlin: Springer, 2001

[14] Henderson, K.L., Barenghi, C.F., Superfluid Couette flow in an enclosed annulus Theoret. Comput. Fluid Dynamics 18, 183-196 (2004)

[15] Schwarz, K.W., Generating superfluid turbulence from simple dynamical rules Phys. Rev. Lett. 49, 283-285 (1982)

[16] Schwarz, K.W., Three-dimensional vortex dynamics in superfluid ${ }^{4} H e$, I. Line-line and line boundary interactions Phys. Rev. B 31, 5782-5804 (1985)

[17] Schwarz, K.W., Three-dimensional vortex dynamics in superfluid ${ }^{4}$ He Phys. Rev. B 38, 2398-2417 (1988) 
[18] Wang, R.T., Swanson, C.E., Donnelly, R.J., Anisotropy and drift of vortex tangle in helium II Phys. Rev. B 36, 5240-5244 (1987)

[19] Peralta, C., Melatos, A., Giacobello M., Ooi A. Global three-dimensional flow of a neutron superfluid in a spherical shell in a neutron star Astroph. Journal 635, 1224-1232 (2005)

[20] Peralta, C., Melatos, A., Giacobello M., Ooi A., Transition between turbulent and laminar superfluid vorticity in the outer core of a neutron star Astroph. Journal 651, 1079-1091 (2006)

[21] Iordanskii, S.V., On the mutual friction in a superfluid, Phys. Rev. Lett. 15, 34-35 (1965)

[22] Anderson, P.W., Considerations on the flow of superfluid helium, Rev. Mod. Phys. 38, 298-310 (1966)

[23] Jou, D., Mongiovì, M.S., Description and evolution of anisotropy in superfluid vortex tangles with counterflow and rotation Phys. Rev. B 74, 054509 (11 pages) (2006)

[24] Tsubota, M., Araki, T., Barenghi, C.F., Vortex Tangle Polarized by Rotation Jour. of Low Temp. Phys. 134, 471-476 (2004)

[25] Barenghi, C.F., Hulton, S., Samuels, D.C., Polarization of superfluid turbulence Phys. Rev. Lett 89, 275301 (4 pages) (2002)

[26] Mongiovì, M.S., Jou, D., A thermodynamical derivation of a hydrodynamical model of inhomogeneous superfluid turbulence Phys. Rev. B 75, 024507 (14 pages) (2007)

[27] Jou, D., Sciacca, M., Mongiovì, M.S., Vortex dynamics in rotating counterflow and plane Couette and Poiseuille turbulence in superfluid Helium Phys. Rev. B 78, 024524 (2008)

[28] Doi, M. and and Edwards S.F., The theory of polymer dynamics, Clarendon Press, Oxford, 1986

[29] Öttinger H.C., Beyond equilibrium thermodynamics Wiley, Hoboken, 2005

[30] Tsubota, M., Barenghi, C.F., Araki, T., Mitani, A., Instability of vortex array and transitions to turbulence in rotating helium II Phys. Rev. B 69, 134515 (12 pages) (2004)

[31] Jou D., Mongiovì, M.S., Phenomenological description of counterflow superfluid turbulence in rotating containers Phys. Rev. B 69, 094513 (7 pages) (2004) DOI 10.1103/PhysRevB.69.094513

[32] Vinen, W.F., Mutual friction in a heat current in liquid helium II. III. Theory of the mutual friction Proc. Roy. Soc. London A240, 493-515 (1957)

[33] Mongiovì, M.S., Jou, D., Sciacca, M., Energy and Temperature of Superfluid Turbulent Vortex Tangles Phys. Rev. B 75, 214514 (10 pages) (2007) DOI 10.1103/PhysRevB.75.214514

[34] Jou, D., Mongiovì, M.S., Sciacca, M., Vortex Density Waves and High-Frequency Second in Superfluid Turbulence Hydrodynamics Phys. Lett. A 368, 7-12 (2007) DOI 10.1016/j.physleta.2007.03.078 
[35] Geust, J.A., Hydrodynamics of quantum turbulence in He II: Vinen's equation derived from energy and impulse of vortex tangle Physica B 154, 327-343 (1989)

[36] Mongiovì, M.S., Jou, D., Superfluid turbulence in rotating containers: phenomenological description of the influence of the wall Phys. Rev. B 72, 104515 (8 pages) (2005) DOI 10.1103/PhysRevB.72.104515

[37] Jou, D., Mongiovì, M.S., Non-Equilibrium Thermodynamics in Counterflow and Rotating Situations Phys. Rev. B 72, 144517 (8 pages) (2005) DOI 10.1103/PhysRevB.72.144517

[38] Sciacca, M., Mongiovì, M.S., Jou, D., A mathematical model of counterflow superfluid turbulence describing heat waves and vortex-density waves Math. Comp. Mod. 48, 206221 (2008) DOI 10.1016/j.mcm.2007.09.007

[39] de Groot, S. R., Mazur, P., Nonequilibrium thermodynamics Amsterdam: North Holland, 1962

[40] Lebon, G., Jou, D., Casas-Vzquez, J., Understanding non-equilibrium thermodynamics Berlin: Springer, 2008

[41] Swanson, C.E., Barenghi C.F., Donnelly, R.J., Rotation of a tangle of quantized vortex lines in He II Phys. Rev. Lett. 50, 190-193 (1983)

[42] Godfrey, S.P., Samuels, D.C., Barenghi, C.F., Linear stability of laminar plane Poiseuille flow of Helium II under a non-uniform mutual friction forcing Phys. Fluids 13, 983-990 (2001) 